\title{
Externe Expertise und lokale Wirklichkeit - Ambivalenzen des lokal-global-Paradigmas im Irak am Beispiel irakischer Frauenorganisationen
}

\author{
Annika Henrizi \\ Center for Conflict Studies, University of Marburg, 35037 Marburg, Germany \\ Correspondence to: Annika Henrizi (annika.henrizi@staff.uni-marburg.de)
}

Received: 28 May 2016 - Revised: 3 November 2016 - Accepted: 2 January 2017 - Published: 26 January 2017

\begin{abstract}
Kurzfassung. External intervention in Iraq has been widely criticised by practitioners and academics: The armed invasion of the US and its allies in 2003 aimed at toppling the Baath Regime and transforming Iraq into a democratic country. While it succeeded in ending the Baath regime, Iraq is far from doing well concerning security, economy and political progress. This situation is often represented as at least partly due to the failure of the US to integrate local perspectives and to pay attention to local needs. Against that backdrop the paper analyses how local actors - in my case non-governmental women's organisations - perceive and interact with international actors and knowledge frameworks. My empirical data show that the assessment of external engagement from a local perspective is far more ambivalent than a binary reading of local vs. external/global as persistent in peace and conflict studies suggests. I break with this binary reading by introducing three concepts to this debate relations, heterogeneity and assessment of external actors. Thus, I aim to draw a more nuanced picture of the role of external expertises for the Iraqi society as it is perceived by women activists.
\end{abstract}

\section{Einleitung}

Der Irak blickt auf eine lange Geschichte des Eingreifens externer Akteure zurück; westliche Mächte sind mindestens seit der britischen Mandatszeit zu Beginn des 20. Jahrhunderts im Irak involviert. Nachdem die USA und ihre Alliierten 2003 in den Irak einmarschierten, um die Diktatur Saddam Husseins zu beenden, erhielt die Diskussion um die Auswirkungen externen Handelns auf lokale Gegebenheiten erneute Brisanz. Im Fokus der Kritik stand dabei nicht nur die Frage nach der Legitimität der Invasion unter Führung der USA, sondern auch der fehlende Einbezug der lokalen Bevölkerung während des Wiederaufbaus (vgl. u.a. Baram et al., 2010; Dodge, 2003; Fürtig, 2010; Slash und Tom, 2011). Die Missachtung und das fehlende Wissen um lokale Gegebenheiten haben bis heute weitreichende Auswirkungen auf viele Bereiche der irakischen Gesellschaft: Die Sicherheitssituation gestaltet sich aufgrund gewaltsamer Konflikte zwischen rivalisierenden (terroristischen) Gruppen - seit 2014 auch des Islamischen Staats (IS) - und fehlender staatlicher Kontrolle weiterhin desolat; die irakische Bevölkerung pro- fitiert kaum von den Ressourcen des Landes; das politische Klima bleibt nach wie vor angespannt, insbesondere auch, weil die USA zu einer Stärkung ethnischer und konfessioneller Zugehörigkeiten beitrugen und so den sozialen Zusammenhalt schwächten.

Im Kontext dieser Kritik erhielt die Situation irakischer Frauen besondere Bedeutung. Zum einen sind Frauen besonders von andauernder Gewalt und wirtschaftlicher Stagnation betroffen. Zum anderen ist die Lage der Frauen besonders brisant, weil sie sich - entgegen den geäußerten Absichten der USA - nach der Invasion weiter verschlechterte (z.B. der Zugang zu Bildung oder häusliche Gewalt): Im Zusammenhang des Vorhabens der USA, die irakische Bevölkerung von der Tyrannei des Saddam-Regimes zu befreien und das Land in eine Demokratie nach westlichem Vorbild zu verwandeln, gewann auch das Thema der Befreiung der ,unterdrückten irakischen Frauen" an rhetorischer und politischer Bedeutung; es wurde teilweise sogar benutzt, um die Invasion im Nachhinein zu legitimieren. Die Arbeit von Nadje Al Ali und Nicola Pratt (2009) schildert ausführlich, dass viele irakische Frauen aber gerade nicht von der Invasion profitierten son- 
dern sich ihre Situation im Gegenteil noch verschlechterte. Das Handeln externer Akteure hatte somit im Irak - gerade aus der Perspektive von Frauen - besonders problematische Auswirkungen (Eigene Interviews; siehe auch Al-Ali und Pratt, 2009; Jawad, 2013).

Vor diesem Hintergrund möchte ich im vorliegenden Artikel der Frage nachgehen, wie externe Expertise von lokalen Frauenorganisationen wahrgenommen wird und wie diese sich gegenüber externen Akteuren, deren Wissensbeständen und Praktiken, positionieren. Die externen Akteure, die ich in den Blick nehme, sind internationale, westliche Akteure, die im Irak agieren, etwa UN-Organisationen oder internationale und nationale Organisationen aus dem europäischen und amerikanischen Raum. Die problematischen Auswirkungen für Frauen legen zunächst nahe, dass lokale Aktivistinnen externen Akteuren geschlossen kritisch gegenüber stehen. Meine empirischen Daten zeigen jedoch, dass die Schilderungen und Erfahrungen der Frauen wesentlich heterogener sind, als eine binäre Gegenüberstellung von ,extern“ und ,lokal“ suggeriert. Dass die Realität in (Postkonfikt-)Gesellschaften hybrid ist, und „lokale“" (ebenso wie globale) Räume durch Beziehungen konstituiert, ist mittlerweile eine breit geteilte Ansicht. Umso erstaunlicher ist es, dass - auch in der Friedensund Konfliktforschung (FuK) - ebensolche Dichotomien auf theoretischer Ebene oft aufrecht erhalten werden. Darüber hinaus bestehen auch in der Praxis des Handelns internationaler Akteure (teilweise jedoch auch in jener lokaler Organisationen) und ferner nicht zuletzt in empirischen Analysen dichotome Konzepte fort. Ich argumentiere hier, dass die Vernachlässigung von Heterogenität Teil eines Raumverständnisses ist, welches Relationalität nicht systematisch anerkennt.

Der vorliegende Artikel möchte deswegen einerseits implizite, dichotome Konzepte innerhalb der FuK hinterfragen und Vorschläge für eine Auseinandersetzung der FuK mit anderen Disziplinen, die sich per se auch theoretisch mit Raum beschäftigen, insbesondere der Geographie, machen. Andererseits zeigt der Artikel mit einer fundierten empirischen Analyse für geographische Debatten auf, wie ein relationales Raumverständnis gerade auch im arabischen Raum fruchtbar gemacht werden kann und welche Relevanz ihre raumtheoretischen Debatten auch für andere Fachdisziplinen haben. Dazu führe ich zunächst drei Konzepte ein, deren analytische Relevanz sich vor allem im Kontrast zum derzeitigen Verständnis des Verhältnisses von lokal und extern in der FuK ergibt: Dort wird das Lokale oft weiterhin als abgegrenzter Ort verstanden und einseitig romantisiert. Damit werden $B e$ ziehungen, die - einem relationalen Verständnis folgend Räume mitkonstituieren, vernachlässigt. Zweitens sind weder lokal noch international agierende Akteure als homogene Gruppen zu verstehen, sondern von ausgesprochener Heterogenität geprägt; anders als eine dichotome Gegenüberstellung von lokalem als Laien- und externem als Expertenwissen der FuK vermuten lässt. Drittens wird oft eine Bewertung des Lokalen als gut und des Internationalen, Externen als schlecht vorgenommen, die sich empirisch nicht halten lässt, weil sich die Akteurinnen sehr unterschiedlich positionieren und externes Wissen keineswegs nur negativ bewerten. Nach dieser Diskussion führe ich in das Handeln externer Akteure im Irak (vornehmlich seit 2003) ein und erörtere kurz die angewandten Methoden und den Untersuchungsgegenstand. In der empirischen Analyse zeige ich schließlich entlang der Konzepte Beziehungen, Heterogenität und Bewertungen auf, wie lokale Aktivistinnen externe Akteure und Expertisen wahrnehmen und mit ihnen interagieren. Eine solche konzeptionelle Herangehensweise ermöglicht ein differenziertes Bild der Hybridität von Postkonfliktgesellschaften und bietet neue Impulse für Policy-Ansätze.

\section{Vom „schlechten“ Globalen und „guten“ Lokalen - Persistenz von Dichotomien in der interdisziplinären Friedens- und Konfliktforschung}

Mit dem „Local Turn“ (vgl. etwa MacGinty, 2011; Richmond, 2012) wurden die Diskussionen um das Lokale auch in der interdisziplinären FuK prominent. Die Beschäftigung mit dem Lokalen ist Teil einer kritischen Bewegung innerhalb der FuK ${ }^{1}$, welche Theorie und Praxis von „,neoliberalen“, extern induzierten Friedensprozessen kritisiert:
... the overarching neoliberal ideology that mer- ges security and development; "romanticizes the local" as victims or illiberal; builds hollow institu- tions; designs economic life to reproduce assertive capitalism; equates peace with statebuilding; and assumes that interveners have privileged knowled- ge about peace issues. The paradigm is mobilized with a package of transformation policies - an as- semblage construed by academics as the "liberal peace" (Pugh, 2013:14).

... Importantly, the liberal peace is not just a framework, it is also a mechanism for the transmission of Western-specific ideas and practices whereby its local agents are not merely compelled to receive, they must also transmit. Thus they become facilitators and enforcers of the liberal peace for municipalities, communities and individuals further down the political chain (MacGinty, 2008:144).

Vertreter_innen des Local Turn fordern, dass lokale Ideen und Akteure von internationalen Akteuren ernstgenommen werden und den Ausgangspunkt für Prozesse der Friedenskonsolidierung bilden. Dabei wird auch von Vertreter_innen ebendieser Richtung anerkannt, dass das Lokale nicht homogen und nicht notwendigerweise positiv ist (vgl. etwa Jabri,

\footnotetext{
${ }^{1}$ Als ,kritische“ Richtung der FuK bezeichnet sich eine Gruppe von Akademiker_innen, die den local turn stark gemacht haben und Ansätze des „liberalen Friedens“ kritisieren (vgl. prominent Oliver Richmond und Roger MacGinty). Der Begriff „kritisch“ wird im Folgenden im Bezug auf diese Richtung verwendet.
} 
2010; MacGinty, 2011). So weist MacGinty eindrücklich auf die Gefahr der „Romanticisation of the local“ (MacGinty, 2011:51) hin und legt dar, inwiefern die Realität indes von Hybridität geprägt ist: „We are all hybrids. Our polities, societies, and economies are the rsult of long procesesses of hybridisation“ (MacGinty, 2011:1). Während jedoch in anderen Disziplinen wie der Soziologie, Humangeographie und Anthropologie die Konstruktion des Lokalen sowie Relationen zwischen lokal und global schon länger Gegenstand tiefgreifender Analysen ist - in der Geographie spätestens seit Swyngedouw (1997) in den 90er Jahren - hat sich die Kritik innerhalb der FuK zunächst mehr auf die Praxis der Friedenskonsolidierung und empirische Phänomene beschränkt, ohne die Konstruktion des Lokalen auf (raum-)theoretischer Ebene zu hinterfragen und in einem zweiten Schritt die Relationalität von Räumen systematisch anzuerkennen. Mittlerweile gibt es auch in der Friedens- und Konfliktforschung Arbeiten, die sich einer raumtheoretischen Perspektive bedienen um Friedensräume und Räume der Gewalt zu analysieren (prominent dazu Björkdahl und Buckley Zistel, 2016). Ich möchte zu Stärkung einer solchen Perspektive beitragen, in dem ich ein relationales Verständnis von Raum vorschlage, um das Verhältnis von lokalen Akteuren und externen Expertisen bzw. Akteuren näher zu beleuchten.

Einem relationalen Raumbegriff folgend, sind Räume nicht gegeben, sondern konstituiert und sozial produziert (vgl. dazu Harvey, 2005; Lefebvre, 1991; Massey, 1999). Objekte, Menschen, Scales oder Diskurse existieren demnach in Beziehungen zueinander und konstituieren so Raum: Prozesse laufen nicht in Räumen ab, sondern konstituieren erst einen Raum. Weiterhin kann Raum nicht ohne die zeitliche Dimension verstanden werden. Raum ist in diesem Sinne kein starres Konstrukt, sondern wandelbar (Harvey, 2005:94ff.). Wenn das Räumliche als durch soziale Beziehungen konstituiert betrachtet wird, dann ist ein Ort (place) eine bestimmte Artikulation dieser Beziehungen (Massey, 1994:5). Ein solcher Raumbegriff bietet ein alternatives Verständnis von lokalen und externen Räumen als wechselseitig verbunden. Im Folgenden möchte ich drei Versionen des binären Raumverständnisses in der Friedens- und Konfliktstudien sowie empirischen Studien aufzeigen und auf Basis meiner empirischen Daten vorschlagen, wie diese sich mit Hilfe eines relationalen Raumbegriffs aufbrechen lassen.

\subsection{Beziehungen}

Zunächst basieren Vorstellungen des Lokalen und Globalen innerhalb der FuK häufig auf einem absolutistischen Raumverständnis welches lokal und global nicht nur inhaltlich, sondern auch konzeptionell binär gegenüberstellt. Während das Lokale implizit als abgrenzbarer Ort im Sinne eines Territoriums verstanden wird auf welches das Globale einwirkt, gilt das Globale und Internationale - das Externe - als flieBend, und nicht an einen bestimmten geographischen Ort gebunden (vgl. dazu ausführlich Buckley-Zistel, 2016; Henri- zi, 2015). Kritiker_innen des liberalen Friedens werfen internationalen Akteuren auf praktischer Ebene genau das vor: nämlich vermeintlich universale Ansätze und Programme zu initiieren, die lokalen Gegebenheiten nicht gerecht werden. Aber auch ebendiese kritischen Ansätze brechen die Dichotomie von lokal und global nicht vollständig (und insbesondere nicht bezüglich der inhärenten Raumvorstellungen) auf. Gerade in Konzepten, die sich mit Hybridität befassen (vgl. etwa MacGinty, 2011; Richmond, 2009) werden lokale und internationale Akteure und Räume weiterhin als getrennte Einheiten postuliert, die sich dann ,,vor Ort“ mischen. Die wechselseitige Konstitution von Akteuren und Räumen wird so auf konzeptioneller Ebene weiter verkannt.

Folgt man dagegen einem relationalen Raumverständnis, ist der lokale Raum (und die in ihm verhandelten Wissensbestände und Praktiken) nicht gegeben, sondern durch Beziehungen konstituiert:

If space is a product of practices, trajectories, interrelations, if we make space through interactions at all levels, from the (so-called) local to the (socalled) global, then those spatial identities such as places, regions, nations, and the local and the global, must be forged in this relational way too, as internally complex, essentially unboundable in any absolute sense, and inevitably historically changing (Massey, 2004:5).

Ebenso können globale und internationale - externe Praktiken als relational, als hergestellt durch Beziehungen, konzeptualisiert werden. Auch sie werden auf unterschiedlichsten Scales konstituiert und treten an verschiedenen Orten in Erscheinung. Doreen Massey betont in ihrem Konzept eines relationalen Raums die Bedeutung von Identitäten; ein relationaler Begriff von Raum ist demzufolge eng mit einem relationalen Verständnis von Identität verknüpft. Individuen sind somit keine isolierten Einheiten mit einer festgelegten Identität. Vielmehr entstehen auch Identitäten nur durch Interaktion. Ebenso sind räumliche Identitäten wie lokal und global immer das Produkt von Beziehungen, die weit über den Ort hinausreichen können. Das Lokale kann zwar einen geographischen Ort meinen, jedoch nicht auf ihn reduziert werden, da er immer auch Beziehungen zwischen Menschen, Diskursen und Gütern einschließt (Massey, 2004:5f, 1991a:28, 1991b:271). „Grundsätzlich werden mit einem relationalen Raumbegriff Beziehungsgeflechte zwischen verschiedenen Dimensionen des Raumes ins Zentrum gestellt. Sie betreffen Merkmale wie abstrakt und konkret, räumliche Maßstabsebenen, Materialität, Symbolik, Diskurse, Vorstellungen oder soziale Beziehungen" (Kemper und Vogelpohl, 2011:24f.; s. dazu auch Harvey, 2009:133ff.; Herv.A.H.).

Wenn Räume als durch soziale Beziehungen konstituiert sind, sind sie, so Massey, auch unweigerlich von Macht durchzogen:

This would imagine the spatial as a sphere of the juxtaposition, or co-existence, of distinct narrati- 
ves, as the product of power-filled social relations; it would be a view of space which tries to emphasize both its social construction and its necessarily power-filled nature (Massey, 1999:21f).

Für die Frage der Wahrnehmung externer Expertise durch lokale Akteure im Irak ist die Frage von Macht besonders bedeutsam.

\subsection{Heterogenität}

Auch Wissensbestände und Akteure sowie deren Handlungsweisen werden oft binär betrachtet: Lokale und Internationale Akteure bleiben als gegensätzliche Kategorien bestehen (siehe etwa MacGinty und Richmond, 2013; Richmond, 2012). Kritische Ansätze erkennen zwar an, dass die Realität zunehmend von Hybridität geprägt ist (vgl. dazu prominent MacGinty, 2011), die inhärenten binären Raumkonzeptionen werden jedoch nicht hinterfragt. Damit wird auch verkannt, dass Akteure nicht nur im Raum agieren, sondern selbst Teil der Konstitution des Raumes sind: Der lokale Raum ist damit, ebenso wie die Gruppe der sog. lokalen Akteure - ebenso wie die der externen - die ihn mitkonstituiert, also nicht homogen, sondern von Heterogenität geprägt. Raum und Multiplizität sind wechselseitig aufeinander bezogen; wenn Raum aus Beziehungen konstituiert ist, dann herrscht in ihm Pluralität (Massey, 1999:22f; vgl. auch Pierce et al., 2010). Wenn ich also in diesem Beitrag von lokalen Akteuren spreche, dann meine ich Akteure, die sich selbst als lokal bezeichnen und sich ganz überwiegend innerhalb des Irak und immer mit engem Bezug zur irakischen Gesellschaft engagieren. Als externe Akteure bezeichne ich solche Akteure, die für Organisationen arbeiten, deren Hauptsitze außerhalb des Irak liegen, meist temporär im Irak arbeiten und stark auf internationaler Ebene agieren. Ich benutze die Begriffe von lokal und extern um Akteursgruppen benennen zu können, dennoch verfolge ich ein Verständnis, dass Heterogenität und Beziehungen zwischen Akteuren anerkennt; keine der beiden Gruppen ist als homogene Gruppe zu verstehen, die isoliert von anderen besteht.

Auch im Bezug auf Wissensbestände wird Heterogenität vernachlässigt: Lokalen Akteuren werden insbesondere solche Wissensformen zugeschrieben, die als "situiert“ oder „Laienwissen“ bezeichnet werden (Haughton et al., 2015:377). Sie haben scheinbar nur das Wissen um die konkreten Gegebenheiten eines bestimmten Ortes (des Lokalen). Demgegenüber wird ,globalen“" Akteuren ein Expertenwissen zugeschrieben, das unabhängig von konkreten Orten existiere und somit auch universell auf jeden Ort übertragbar sei. Dieser Logik folgend, wird lokales Wissen mit Laienwissen oder situiertem und externes Wissen mit Expertenwissen gleichgesetzt (kritisch dazu Haughton et al., 2015:277ff.). In Konflikt- und Postkonfliktgesellschaften, wie dem Irak, kommt es durch das Handeln externer - meist westlicher Akteure zu Konfrontationen zwischen lokalen und externen
Akteuren und deren Handlungsweisen. Vertreter des Local Turn fordern eine Emanzipation des Lokalen und damit verbunden eine Neuorientierung bisheriger Policy-Ansätze in Konflikt- und Postkonfliktgesellschaften. Damit soll einem oberflächlichen Machttransfer auf lokale Akteure und der technokratischen Ausrichtung internationaler Einsätze entgegengewirkt werden (vgl. etwa MacGinty, 2011; Richmond, 2010). So stellt MacGinty in seinem Artikel zu „Indigenous Peacemaking Versus The Liberal Peace“ (2008) indigenes, lokales und westlich geprägte Formen der Friedenskonsolidierung gegenüber:

This piece considers the compatibility of traditional, indigenous and customary forms of peacemaking in civil wars with the currently dominant Western form of peace-making and peace support, often described as the 'liberal peace' (MacGinty, 2008:139).

Obwohl ebensolche Ansätze wichtig für die Diskussion um internationale Politik in Postkonfliktkontexten sind, bergen sie weiterhin das Risiko, lokales und externes Wissen dichotom gegenüberzustellen und Heterogenität zu vernachlässigen.

Analog zu Haughtons et al. (2015) Analyse von früheren Auffassungen der Bewertung des Lokalen in der geographischen Forschung zum Management von Hochwasserrisiken werden auch in der FuK vereinfachte Bilder von lokalem, situierten und externem (hier nicht staatlichem sondern international-westlichen) Wissensbeständen kreiert:

This emerging body of work has helped move our understanding beyond early accounts of local knowledge, which sometimes presented a romanticised view of the role of communities in creating and stewarding situated knowledge, while tending to present an oversimplified version of state knowledge as its antithetical opposite, a form of remote, expert knowledge that overrides local knowledge (Haughton et al., 2015:377).

Während die Geographie hier über die theoretischen Werkzeuge verfügt, sind es insbesondere empirische Studien, welche ein tieferes Verständnis lokaler Wissensformen fördern können, an denen es zum Teil fehlt. In der FuK existieren zahlreiche ebensolcher Studien; es fehlt aber an einer systematischen Auseinandersetzung mit Differenzen innerhalb dieser Wissensformen - auch hier können relationale Raumkonzeptionen weiterführen.

Es wäre also sinnvoll in den genannten Ansätzen der FuK die impliziten Raumvorstellungen sichtbar zu machen und so eine systematische Auseinandersetzung mit Raum zu ermöglichen. Wenn lokale Räume als Orte verstanden werden, die schon aufgrund ihrer sozialen Konstitution heterogen sein müssen, dann sind auch lokale Wissensbestände und Praktiken aus einer Vielzahl von Einflüssen entstanden und formen gleichsam lokale Räume: 
This is a notion of place where the specificity (local uniqueness, a sense of place) derives not from some mysthical internal roots nor from a history of relative isolation - but precisely from the absolute particularity of the mixture of influences found together there (Massey, 1999:22).

Das Lokale ist nach einem solchen Verständnis also kein isolierter Raum, in dem Menschen und ihre Handlungsweisen isoliert existieren, sondern immer von Heterogenität geprägt und wandelbar.

\subsection{Bewertung}

In der kritischen FuK folgt diesen beiden binären Konstruktionen eine dritte Ebene, nämlich die der Bewertung ebendieser Wissensformen und Akteure als gut (lokal) und schlecht (extern). Externes Wissen wird, wie oben erläutert, als liberal, technokratisch und fern lokaler Gegebenheiten deklariert und damit negativ bewertet (etwa MacGinty und Richmond, 2013, Zitat unten). Gerade im Bezug auf Zivilgesellschaft betonen kritische Ansätze die negativen Auswirkungen, welche internationale NGOs auf lokale Zivilgesellschaft haben können:

When a "crisis" is discovered by the "international community", international NGOs are part of the real "invasion". Hundreds of international organizations are present in the capital, occupying a space no longer available to local actors, ..., which impede local organizations from functioning properly (Pouligny, 2005:501).

Lokale Organisationen würden, so die Kritik, durch einseitige Abhängigkeitsverhältnisse jeglicher Handlungsmächtigkeit beraubt (Cubitt, 2013; Pouligny, 2005). Obwohl die Kritik sicher teilweise berechtigt ist, zeichnet sie ein zu einfaches Bild der komplexen Verhältnisse in (Post-)Konfliktgesellschaften. In Abgrenzung dazu unterstellen Wissenschaftler_innen lokalem Wissen und Handeln positive, eher friedliche, Effekte (Pouligny, 2005; MacGinty, 2008):

First, in post-conflict reconstruction, indigenous and autonomous forms of local association are more legitimate and appropriate for broader citizen representations than the civil society bourgeoisie constructed from outside (Cubitt, 2013:92).

Allerdings wird zunehmend reflektiert, dass auch das sog. Lokale gewaltfördernd und diskriminierend sein kann und nicht unbedingt zur friedlichen Transformation von Gesellschaften führt: ,,... that local actors and contexts can be partisan, discriminatory, exclusive and violent" (MacGinty und Richmond, 2013:270; siehe auch Hellmüller und Santschi, 2014: Is Local Beautiful?). Obwohl sich die Debatte insgesamt stetig weiterentwickelt, bleiben solche Einsichten aber eher anekdotisch, ohne die häufig impliziten normativen Bewertungen von lokal als gut und extern als schlecht auch auf theoretischer Ebene zu durchbrechen. Gerade in den Annahmen darüber, wie lokale Akteure externes Handeln und Wissen beurteilen, treten Bewertungen besonders klar hervor:

It also is easy to forget that a local perspective of the international (a perspective commonly repeated across both the authors' years of fieldwork) is that it is also endemically dysfunctional, contextually insensitive, disrespectful and distant, unaccountable, interest-based, normatively biased, ideologically fixed, mercenary in its naturalisation of capitalism and unwilling to address inequality or the historical injustices stemming from colonialism. (MacGinty und Richmond, 2013:272).

Ein solches binäres Bewertungsschema greift zu kurz. Im Gegensatz zu MacGintys und Richmonds Erfahrungen spiegeln meine empirischen Daten ein wesentlich ambivalenteres Bild wider: Lokale Akteure bewerten externes Wissen nicht nur einseitig negativ, sondern sowohl positiv wie auch negativ und in beiden Fällen sehr differenziert und divers. Vielmehr positionieren sie sich gegenüber externem Wissen strategisch auf einer breiten Achse von Anerkennung und Ablehnung - je nachdem wie nützlich und relevant bestimmte Themen und Wissensbestände für sie sind. Um diesen ambivalenten Ausprägungen gerecht zu werden, müssen Dichotomien über die empirischen Daten hinaus auch auf theoretischer Ebene aufgebrochen werden. In Anlehnung an Haughton, Bankoff und Coulthard möchte ich im empirischen Teil erörtern, wie externes und lokales Wissen (aus der Perspektive lokaler Akteure) interagieren und wie das oben geschilderte binäre Konzept eine differenzierte Bewertung der multiskalaren Beziehungen verhindert: „,.. expert and lay knowledge intersect in concrete contexts, challenging accounts constructed around false binaries and an incomplete appreciation of the complexity of knowledge formation practices" (Haughton et al., 2015:377).

\section{Das Handeln externer Akteure im Irak - Kontext}

\subsection{Irak allgemein}

Der Irak schaut auf eine lange Phase der Einmischung externer Akteure ${ }^{2}$ zurück. Auch und insbesondere die Situation irakischer Frauen ist von diesem Handeln beeinflusst. Wie Aktivistinnen heute externe Expertise wahrnehmen und sich dazu positionieren, ist eng mit der Geschichte internationalen Engagements im Irak verknüpft. Ich werde daher im Folgenden kurz auf einige jüngere Entwicklungen im Irak, die

\footnotetext{
${ }^{2}$ Externe Akteure, welche die Situation im Irak beeinflusst haben sind keinesfalls nur ,westliche“ Akteure. Da sich der vorliegende Artikel jedoch auf diese Akteure bezieht, lege ich auch hier den Fokus auf das Handeln europäischer bzw. US-amerikanischer Akteure
} 
für meine Forschung (mit Bezug auf externes Handeln) relevant sind, eingehen, um dann die spezifischen Auswirkungen für Frauen zu erläutern. Die aktuelle Situation im Irak ist jedoch keinesfalls nur auf westliches Handeln zurückzuführen sondern Ergebnis einer langen Geschichte von gewaltsamen Konflikten, Bürgerkriegen und Terrorismus, in die lokale ebenso wie regionale und internationale Akteure verstrickt sind.

\section{Überblick 1: Untersuchte externe und lokale} Akteursgruppen

Bereits zu Beginn des 20. Jahrhunderts war das Land vom Handeln westlicher Kolonialmächte - insbesondere Großbritannien - beeinflusst. Auch nach dem Ende der britischen Mandatszeit und der Gründung als moderner Staat 1932 haben internationale Akteure immer wieder in das irakische Geschehen eingegriffen. Demokratie stand schon seit mehreren Dekaden auf der Agenda arabischer Staaten und wurde insbesondere seitens der progressiven säkularen Opposition in den 60er und 70er Jahren gefordert; westliche Mächte wie die USA und Großbritannien unterstützen stattdessen autokratische Regime, da sie der Auseinandersetzung mit der Sowjetunion größere Priorität einräumten (Rahbek, 2005:7). Mit der irakischen Invasion in Kuweit 1990 ändert sich die Haltung des Westens gegenüber dem Irak. Nach vergeblichen Bemühungen, Saddam Hussein zum Abzug zu bewegen, wurde die irakische Armee durch die Operation „,Desert Storm“ militärisch aus Kuweit zurückgedrängt. Als offizielle Beendigung des Krieges verhängt die UN weitreichende Sanktionen, welche aufgrund der verheerenden Auswirkungen für die Lebensbedingungen der irakischen Zivilgesellschaft (insbesondere Kinder und Frauen) vielfach kritisiert wurden (Fürtig, 2004; Al-Jawaheri, 2008).

Neue Brisanz erhielt das Handeln westlicher Akteure nach 2003. Der Fokus meines Artikels liegt auf ebendieser Zeitspanne: Unter dem Vorwand, der Irak besitze Massenvernichtungswaffen und sei nicht bereit mit dem Sicherheitsrat zu kooperieren, formte die USA 2003 eine „Koalition der Willigen“ (bestehend aus 30 Staaten), die in den Irak einmarschierten, um das Regime von Saddam Hussein zu stürzen und das Land zu demokratisieren. Dabei fungierten die USA und Großbritannien laut UN-Sicherheitsrat als Besatzungsmächte (Kbashi et al., 2014). Nicht nur die direkte Invasion, sondern auch wie die USA und ihre Alliierten Demokratisierung und Wiederaufbau planten und durchführten, ist durch akademische Forscher_innen (vgl. etwa Al-Ali und Pratt, 2009; Coyne, 2010; Dodge, 2003; Isakhan, 2014; Jawad, 2013) ebenso wie durch internationale und lokale NGOs (etwa Kbashi et al., 2014) vielfach kritisiert worden. Die Folgen der Invasion sind dabei hochkomplex und betreffen Menschen in sehr unterschiedlicher Weise; Berichte darüber sind immer auch von der gelebten Realität von Menschen und starker Meinungsbildung geprägt (Isakhan, 2014). Ich möchte im Folgenden zwei Sichtweisen aufgreifen, die für das Thema der Verhandlung lokalen und externen Wissens besonders relevant sind: Zum einen die (vornehmlich politik-)wissenschaftlichen Analysen zu den Folgen externen Handelns im Irak für Frauen und zum anderen im empirischen Teil die Einschätzungen meiner Interviewpartnerinnen selbst.

Die innerhalb der Forschung angeführten Gründe für das Scheitern der Besatzer und für die anhaltende Gewalt im Irak reichen dabei von schlechter Planung und Zeitmanagement sowie fehlender Strategie zu unzureichenden PolicyAnsätzen und praktischer Inkompetenz (vgl. Al-Ali und Pratt, 2009; Coyne, 2010; Dodge, 2003). Gemeinsam ist den oben und im Folgenden genannten kritischen Ansätzen jedoch, dass sie den Alliierten vorwerfen, lokale Gegebenheiten zu missachten. Diese Perspektive wird auch von vielen der von mir interviewten irakischen Frauenorganisationen vertreten; sie betonen insbesondere die fehlende Kenntnisse über kulturelle und soziale Strukturen und die mangelnde Konsultation der Bevölkerung vor Ort (Eigene Interviews; vgl. auch Kbashi et al., 2014). Die Alliierten erzwangen einen schnellen Staatsbildungsprozess und die Installation einer Demokratie nach westlichem Vorbild, ohne die Realität vor Ort begriffen und ernst genommen zu haben. Vorgefertigte Lösungen wie freie Marktwirtschaft und die Bildung demokratischer Institutionen führten zur Entstehung von Programmen und Strukturen (bspw. Prozess der Verfassungsbildung, kommunale Regierungen, militärische Strukturen), welche nur unzureichend von der irakischen Bevölkerung legitimiert waren und zu einer Vielzahl an gewalttätigen Auseinandersetzungen und Problemen führten.

Der Prozess der De-Baathifizierung hatte bspw. eine Lahmlegung der gesamten Verwaltung zur Folge; arabische Sunnit_innen gewannen den Eindruck, Verlierer_innen der Invasion zu sein (Fürtig, 2010:130). Die Auflösung der Armee stürzte viele Männer in die Arbeitslosigkeit und bereitete so den Nährboden für radikale Gruppierungen. Ehemalige Milizen wurden zur neuen Basis des Heeres (Fürtig, 2010:130; Isakhan, 2014). Weiterhin belebten die Alliierten die ,Teile- und Herrsche“-Strategie entlang ethnischer und konfessioneller Grenzziehungen der Briten wieder: Jede_r, die/der sich am politischen Transformationsprozess beteiligen wollte, musste sich einer konfessionellen bzw. ethnischen Gruppe zuordnen. Diese Reduzierung der Iraker auf Konfession und Ethnie ließ säkularen Bewegungen keine Chance und verstärkte das innerirakische Gewaltpotential (Fürtig, 2010:131; Kbashi et al., 2014).

Insgesamt legte das erfolglose Krisenmanagement zu Beginn der Invasion den Grundstein für die tiefen Gräben zwischen lokaler Bevölkerung und externen Akteuren: Die irakische Bevölkerung nahmen die USA und die ausgeübte Direktverwaltung nicht als Befreier, sondern als Besatzer wahr (Fürtig, 2010:130). Als Reaktion auf Demokratisierung und Liberalisierung entstanden machtvolle Gegendiskurse und Widerstandspraktiken, welche den Besatzern reines Eigeninteresse vorwarfen und in Abgrenzung zu westlichen Werten 
Konservatismus und Islamisierung weiter förderten (Dodge, 2003; Slash und Tom, 2011:195ff.). Der Irak ist insofern ein Paradebeispiel für die verheerenden Auswirkungen einer technokratisch ausgerichteten Friedenskonsolidierung, die lokale Gegebenheiten missachtet.

\subsection{Die Situation irakischer Frauen/genderspezifische Auswirkungen externen Handelns}

Die Wahrnehmung und Positionierung lokaler Frauenorganisationen gegenüber internationalen Akteuren heute ist eng mit den historischen und anhaltenden Handlungsweisen externer Mächte im Irak verknüpft: Zum einen haben die oben genannten problematischen Entwicklungen in der Folge der US-Invasion 2003 Frauen besonders hart getroffen und ihre Lebensbedingungen weiter verschlechtert (Eigene Interviews; Al-Ali und Pratt, 2009; Jawad, 2013). Aktivistinnen bekommen diese Probleme nicht nur selbst zu spüren, sondern werden auch in der täglichen Arbeit mit Frauen damit konfrontiert (Eigene Interviews). Zum anderen sind Frauenrechte und Genderbeziehungen im Irak, mindestens seit der britischen Besatzungszeit, ein hart umkämpftes Thema - sowohl unter lokalen als auch zwischen lokalen und externen Akteuren. Vielfach sind sie dabei zum Spielball politischer Rhetorik geworden (Efrati, 2011). Diese historischen und anhalten Prozesse des Verhandelns von Frauenrechten prägen das gesellschaftliche und politische Klima, in dem Frauen an diesen Themen arbeiten. In Anlehnung an Rohde und Fischer-Tahir verstehe ich die Invasion von 2003 nicht als Stunde Null in der Geschichte des Iraks (und irakischer Frauen), sondern als einen erneuten „Bruch“, der jedoch nur vor dem ,long duree“ der Entwicklung sozialer Beziehungen und gesellschaftlicher Entwicklung im Irak zu verstehen ist (Rohde und Fischer-Tahir, 2015). Diese Perspektive wurde auch von den meisten meiner Interviewpartnerinnen vertreten (Eigene Interviews; Shadow-Report, 2014).

Verglichen mit anderen Ländern der Region war die gesellschaftliche Stellung von Frauen im Irak - insbesondere die rechtliche und wirtschaftliche Lage aber auch die Teilhabe an Bildung bis zur Machtübernahme Saddams 1979 bzw. sogar bis in die Anfangsjahre der Diktatur - relativ gut. Dennoch wurden Frauenrechte immer wieder anderen Themen und Prioritäten untergeordnet. Bereits die britischen Besatzer ,verkauften“ Frauenrechte, in dem sie lokalen Eliten und Stammesführern zusicherten, sich nicht in Angelegenheiten des Familienrechts einzumischen und so deren Loyalität gewannen. Genderbeziehungen und Frauenrechte verbesserten sich dennoch in den ersten Jahren des Baath Regimes: Die Teilhabe von Frauen an Bildung und Wirtschaft nahm zu und das irakische Zivilstandsrecht von 1958 galt als eines der fortschrittlichsten der Region. Gegen Ende der BaathEpoche war das Regime erneut gezwungen, paternalistische Privilegien den Scheichs zu überlassen, um sich so deren Loyalität - und damit Macht - zu sichern (Kamp, 2005:307; Rohde, 2010:74).
Die Invasion der Alliierten 2003 sowie die oben genannten problematischen Auswirkungen der Demokratisierungsbemühungen und des Wiederaufbaus haben innerhalb des Iraks eine Situation geschaffen, die sich kaum als „PostKonflikt" ${ }^{* 3}$ beschreiben lässt, insbesondere nicht aus einer Genderperspektive. Viele Probleme wie Sicherheit, Gewalt, fehlende Infrastruktur und wirtschaftlicher Stillstand haben spezifische Auswirklungen für Frauen. Sie sind Opfer von Gewalt zwischen rivalisierenden Gruppen, aber auch von zunehmender häuslicher Gewalt geworden. Auch ist es vielen Frauen nicht möglich oder nicht erlaubt, sich alleine im öffentlichen Raum zu bewegen. Aktivistinnen berichten auBerdem von Belästigungen und Angriffen, wenn sie sich in westlicher Kleidung oder ohne Kopftuch auf die Straßen wagen. Sie leiden hier unter der zunehmenden Islamisierung und Konservatismus - Phänomene, die zumindest teilwiese als Reaktion auf die proklamierten westlichen Werte verstanden werden (Eigene Interviews; Isakhan, 2014:145; AlAli, 2008). Frauen und insbesondere Witwen erleben extreme wirtschaftliche Einschränkungen und die Teilhabe von Frauen an Bildung ist in den letzten Jahren stark zurückgegangen. Außerdem sind Frauen mit spezifischen Problematiken wie Zeitehen, Kinderehen und Eheschließungen außerhalb staatlicher Behörden sowie Gewalt gegen Frauen konfrontiert (Shadow Report, 2014; Al-Ali, 2008; Kamp, 2005; eigene Interviews).

Auch in Bezug auf Frauenrechte führte das Handeln der Alliierten zu Problemen. Trotz wiederholter Beteuerungen im Vorfeld der Invasion, irakische Frauen ,retten“ zu wollen, ordneten sie Frauenrechte anderen Prioritäten unter: Um Konflikte zu vermeiden und die Loyalität religiöser Führer zu sichern, stimmten sie der Eingliederung des Artikels 41 in die irakische Verfassung zu. Der Artikel ermöglicht es, Familienrechtsangelegenheiten nach dem jeweiligen Glaubensgrundsätzen zu handhaben und überlässt die zivilen Rechte irakischer Frauen so der Verantwortung religiöser Führer. Der Aufschrei von Frauenaktivistinnen, die fürchteten zu verlieren, wofür sie jahrelang gekämpft hatten, führte zu einer zeitlichen Suspension des Artikels, der jedoch bis heute nicht vollends entfernt wurde (Al-Ali und Pratt, 2009; Efrati, 2011:16).

Trotz der wiederholten Rückschläge und der anhaltend schwierigen Situation von Frauen haben Irakerinnern seit 2003 immer wieder die Beteiligung an Wandlungsprozessen und die Verbesserung der rechtlichen und lebensweltlichen Situation von Frauen gefordert und sich vielfach in Frauenorganisationen organisiert. Wie ich im empirischen Teil aufzeigen werde, ist das Verhältnis zu externen Akteuren (direkt zu Gebern und indirekt die Wahrnehmung betreffend) trotz

\footnotetext{
${ }^{3}$ Feministische Ansätze innerhalb der interdisziplinären FuK betonen, dass eine Strenge Einteilung in Konflikt und Postkonfliktgesellschaften den Erfahrungen von Frauen nicht gerecht wird, da diese auch nach der Beendigung offizieller Kriegshandlungen Gewalt ausgesetzt sind (Porter, 2007:63ff.)
} 
der schwierigen Historie heute sehr ambivalent und lässt sich kaum in ein dichotomes Verständnis vom guten Lokalen und schlechten Externen pressen. Einerseits sind Aktivistinnen täglich mit den negativen Auswirkungen des Handeln externer Akteure auf die Situation von Frauen konfrontiert: Während die Meinungen der Frauen gegenüber der Notwendigkeit einer Invasion sehr divers sind, stößt die Art und Weise wie diese durchgeführt wurde weitgehend auf Kritik. Zudem sehen sie sich gerade aufgrund der US-Rhetorik, die sich die „Rettung der irakischen Frauen“ auf die Fahnen schrieb, permanent der Kritik ausgesetzt, westliche Agenden zu verfolgen. Dennoch sehen sie im Regimewechsel andererseits auch eine Chance, sich zu engagieren und arbeiten teilweise schon viele Jahre mit westlichen Geberorganisationen zusammen.

\section{Methoden und Untersuchungsgegenstand}

Wie bereits erörtert, hat das Handeln externer Akteure für Frauen und deren gesellschaftliche Stellung besondere Bedeutung. Deshalb sind die Frauen, die sich engagieren werden und aktiv mit externen Akteuren interagieren, der Fokus meiner Analyse. Ihre Arbeit im Bereich Genderbeziehungen und Frauenrechte ist stark durch externe Akteure und Anfeindungen auf lokaler Ebene geprägt und nur vor diesem Hintergrund zu verstehen.

Der vorliegende Artikel ist im Kontext einer Untersuchung der Handlungsweisen von zivilgesellschaftlichen Frauenorganisationen im Irak (mit Fokus auf Bagdad und den Zentralirak) entstanden. Hierbei geht es insbesondere auch um ihre Haltung gegenüber internationalen Akteuren. Ich habe in der Untersuchung einige Analyseschritte der Grounded Theory (insbesondere nach Charmaz, 2006) genutzt, weil diese für meine Forschung besonders hilfreich waren, ohne mich jedoch der Grounded Theory als Methodologie - auch im Sinne einer Theoriegenerierung - zu verschreiben. Mein Ziel war es, eine „dichte Beschreibung“ (Charmaz, 2006:14) zu erarbeiten und so ein tiefes Verständnis der Denk- und Handlungsweisen irakischer Aktivistinnen ${ }^{4}$ zu erlangen und Bedeutungen zu rekonstruieren. In der Datenerhebung habe ich mich auf sechs Organisationen (drei davon besonders intensiv) beschränkt, mit denen ich tiefgehender arbeiten konnte. Während meiner Feldforschung habe ich 29 ,intensive“ Interviews (Charmaz, 2006:25) und eine Gruppendiskussion mit irakischen Aktivistinnen und Mitarbeiterinnen internationaler NGOs geführt. Außerdem habe ich als teilnehmende Beobachterin an vier Konferenzen

\footnotetext{
${ }^{4}$ Die Bezeichnung „Aktivistin“ kann im Irak - wie in anderen Ländern der Region auch (vgl. ausführlich Al-Ali, 2003) - sehr unterschiedliche Bedeutungen haben und reicht im Selbstverständnis der Frauen von humanitären Aufgaben bis zu politischem Aktivismus. Ich verwende den Begriff, weil er von meinen Interviewpartnerinnen selbst so verwendet wird, hauptsächlich verstanden als Aktivismus für Frauenrechte; in meiner Untersuchung haben alle Frauen ihr Engagement als über das humanitäre hinausgehend im Sinne eines gesellschaftspolitischen Aktivismus verstanden.
}

und Workshops partizipiert und diverse schriftliche Materialien wie NGO-Berichte und Statements analysiert. Darüber hinaus habe ich unterschiedlichste Quellen benutzt, die im Verlauf des Forschungsprozesses zugänglich waren: Insbesondere zufällige Gespräche, Skype-Konversationen, Social Media-Einträge etc. Das ermöglichte mir, kontinuierlich und auf informellerer Ebene mit den Aktivistinnen in Kontakt zu bleiben. Die Daten wurden mittels initialem, axialen und selektiven Kodierens analysiert; außerdem habe ich mich während des gesamten Kodierprozesses des Schreibens von „Memos" (Charmaz, 2006:73ff.) als Methode bedient.

Das Sample der von mir untersuchten Organisationen wurde aus inhaltlichen und forschungspraktischen Gründen gewählt. Um der Frage nach Beziehungen zwischen lokalen und externen Akteuren nachzugehen, wurden solche Organisationen einbezogen, die mit internationalen Geldgebern und/oder NGOs zusammenarbeiten. Gleichzeitig dienten mir so internationale NGOs als Mittel der Kontaktaufnahme mit lokalen Akteuren. Weitere Interviewpartnerinnen wurden dann mittels des Schneeballsystems ermittelt. Meine Informantinnen sind Mitglieder lokaler, nichtreligiöser Frauenorganisationen in Bagdad, die im Bereich von Frauenrechten und Genderbeziehungen tätig sind. Alle Organisationen bezeichnen sich selbst als „lokal“; die meisten arbeiten in und um Bagdad, während andere Büros und Zentren über das Land verteilt betreiben. Die Organisationen werden typischerweise von internationalen Geldgebern unterstützt und wurden (mit zwei Ausnahmen) nach 2003 gegründet. Damit ist das Handeln internationaler Akteure im Irak für die Organisationen auf unterschiedlichen Ebenen relevant: In ihrer Funktion als Geldgeber und Partner nehmen sie direkten Einfluss auf Möglichkeiten und Ausgestaltung der Arbeit von NGOs. Darüber hinaus prägen sie den alltäglichen Handlungskontext der NGOs in der irakischen Gesellschaft. Die Daten spiegeln aufgrund des spezifischen Samples jedoch nur einen Teil der irakischen Frauenorganisationen wider, sie bilden in keiner Weise das gesamte Spektrum irakischer $\mathrm{Zi}$ vilgesellschaft und insbesondere nicht irakischer Frauen ab.

Gerade in Anbetracht meiner Forschungsfrage nach der Wahrnehmung „externer Akteure“ durch „lokale Aktivistinnen" ist meine eigene Positioniertheit als Forscherin besonders relevant. Da Machtverhältnisse in Forschungsprozessen kaum aufzuheben sind, schlägt Sultana (2007) vor, sie andauernd zu reflektieren und sichtbar zu machen. Auch Peake und Trotz betonen, das eine solche Reflektion

can strengthen our commitment to conduct good research based on building relations of mutual respect and recognition. It does, however, entail abandoning the search for objectivity in favour of critical provisional analysis based on plurality of (temporally and spatially) situated voices and silences. (Peake und Trotz, 1999:37).

Die Forderung, Selbstreflexion systematisch in die Forschung einzubeziehen ist in der FuK mittlerweile durch 
feministische Forschungen prominent geworden (vgl. etwa Ackerly et al., 2006; Streicher, 2011). In meinem Fall fällt zunächst die Differenz zwischen mir als weißer Frau aus Deutschland und irakischen Aktivistinnen ins Auge. Doch auch hier werden Dichotomien aufgebrochen, da diese Position von anderen Positionen durchkreuzt wird. Das Interesse an der Situation von Frauen und wissenschaftliche bzw. praktische Arbeit in diesem Bereich ist ein verbindendes Moment. Relativiert werden Machtverhältnisse dadurch, dass ich als Doktorandin noch in der Ausbildung bin, während meine Interviewpartnerinnen im Berufsleben stehen und meist ökonomisch besser gestellt sind. Viele von Ihnen sind älter als ich (im Irak ein deutliches Hierarchiemerkmal). AuBerdem stimme ich aufgrund meiner eigenen Erfahrungen Ruth Streicher (2011:69) in der Annahme zu, dass Macht nie einseitig von der Forscherin ausgeübt wird. Gerade weil ich im Feldzugang sehr auf die Mithilfe meiner Partnerinnen angewiesen war, bestimmen sie mit, was ich erfahre und wie. Sowohl Erzählungen als auch Schweigen sind räumlich und zeitlich situiert und entstehen in einem Raum, der unweigerlich von vielseitigen Machtverhältnissen geprägt ist. In diesem Sinne habe ich meine eigene Rolle und den Forschungsprozess in Feldtagebüchern und Memos reflektiert, muss aber in Kauf nehmen dass sie partiell bleiben: ,... findings will always be partial, yet telling stories that would otherwise not have been told." (Sultana, 2007:382) Damit werden aber auch andere Stimmen zum Schweigen gebracht oder überhört.

\section{Wahrnehmung und Wirkung externer Expertise aus lokaler Perspektive}

Der Fokus meiner Analyse liegt nun auf der Frage, wie Aktivistinnen in Frauenorganisationen im Irak externes (westliches) Wissen und Handeln und seine Wirkungen auf lokale Kontexte wahrnehmen. Ich zeige, dass zwischen den unterschiedlichen Akteuren (mit ihren Wissensbeständen und Handlungsweisen) vielfältige Beziehungen bestehen. Kommunikation und Zusammentreffen zwischen lokalen und externen Akteuren sind allerdings keineswegs immer konfliktfrei. Vielmehr sind die wechselseitigen Aushandlungsprozesse von Machthierarchien, gegensätzlichen Vorstellungen und Denkweisen geprägt, die gemeinsam Raum konstituieren. Dabei sind sowohl die Akteure selbst als auch Wissensbestände wesentlich heterogener als ein binäres Verständnis von lokal und extern suggeriert. Auch eine dualistische, schematische Bewertung von gut und schlecht stimmt nicht mit der Wahrnehmung lokaler Akteure überein.

\subsection{Beziehungen}

Der Irak und speziell Bagdad als Raum ist - über den geographischen Ort hinaus - wesentlich durch vielfältige Beziehungen von Menschen, Gütern und Diskursen konstituiert. NGOs als Teil dieses Raums werden entscheidend durch ihre menschlichen, materiellen und diskursiven Austauschbeziehungen zu Geberorganisationen geformt. Zwischen lokalen und internationalen Akteuren existieren materielle Machthierarchien, welche den Ort beeinflussen: Die von mir untersuchten Organisationen sind - wie die meisten säkularen NGOs - auf die Finanzierung durch internationale Geldgeber (etwa internationale NGOs oder UN Agencies) angewiesen, da es sonst kaum Möglichkeiten der Finanzierung gibt. Dadurch müssen sich lokale Organisationen in vielen Aspekten an die Vorgaben und Handlungsansätze westlicher Geber anpassen, um überhaupt Projekte durchführen zu können. Eine der von mir untersuchten Organisationen versuchte 2014 durch ein kleines Hotel eigene Einnahmen zu generieren und damit eine größere Unabhängigkeit von Gebern zu erlangen und Projekte durchführen zu können, die wenig Chancen auf eine Geberfinanzierung haben. Dennoch sind die materiellen Austauschbeziehungen überwiegend von einem einseitigen Abhängigkeitsverhältnis dominiert.

Die diskursive Ebene - welche eng mit der materiellen verbunden ist - wird seitens der Aktivistinnen wesentlich diverser wahrgenommen. Die Frage, wie sich die Beziehung zu westlichen Geberorganisationen gestaltet, beantworten sie auf einer Bandbreite von wenig Handlungsspielräumen und kritischer Haltung bis hin zu einem eher partnerschaftlichen Verhältnis.

Die verbreiteteste Einschätzung lautet, dass Hierarchien zwar fortbestehen, sich die Beziehungen zu Geberorganisationen aber wandeln. Während NGOs zunächst hauptsächlich den von Geberorganisationen vorbestimmten Programmen (zu Themen wie Geschlechtergerechtigkeit, Jugend, Friedensförderung/Dialog) gefolgt sind, verfügen sie mittlerweile über mehr Möglichkeiten, um eigenes Wissen, Ideen und Handlungsansätze einzubringen. Dazu hat zum einen das Scheitern bzw. die Ratlosigkeit internationaler Akteure gegenüber der andauernden Gewalt und des Scheiterns von Programmen, zum anderen aber auch das wachsende gegenseitige Vertrauen beigetragen. NGO-Mitglieder berichten, dass internationale Geber mittlerweile auch lokales Wissen als relevant anerkennen und mehr von der Kompetenz und Vertrauenswürdigkeit der Organisationen überzeugt sind (Eigene Interviews). Auf der diskursiven Ebene ist also aus dieser Perspektive durchaus der Aufbau von Austauschbeziehungen feststellbar.

It has changed. At the beginning yes, everything, almost everything was imposed and almost all local NGOS they were following, but later on they knew what they wanted. Many they still accept imposing agendas, and some no, they refuse. Recently I feel, the last two years now, things have changed, even with the American organizations, they listen (Interview NGO Mitglied, 24. September 2012). 
Eine andere Aktivistin erklärt, dass sie selbst auch thematische Inputs geben und über grössere Mitbestimmungsrechte verfügen:

We are pushing, we are telling the donors what we shall do now. I will give you an example of D1 (donor organisation). They are working in post[conflict] and conflict countries, but we design our projects. Concerning a study trip in Beirut, it was our design, our strategy, our plan to do it like that. And we discussed it, and they agreed, and then we did it (Interview NGO Mitglied, 25. September 2012).

Andere Frauen sehen die Situation kritischer. Insbesondere Frauen, die selbst schon für internationale NGOs gearbeitet haben, betonen, dass lokale Organisationen eher ausführen als selber initiieren und Prioritäten und Trends internationaler Organisationen folgen:

So this, if you track the activities if the NGOs you can see in 2004/2005 there were projects on conflict resolution, 2006/2009 programs on youth and peace building and from 2009 programs on women's issues because of the international NGOs working on that. So you can see all the NGOs now working on woman issues (Interview NGO Mitglied, 4. Mai 2013).

Eine andere Aktivistin erklärte im Interview, dass lokale Organisationen Ideen oft vor internationalen entwickeln, letztere die Bedeutung oftmals aber erst später realisieren und anerkennen:

I am telling you about when in 2004 when we made our proposal to International Organisations and the UN about capacity building about the NGOs, nobody listened to us, ..., but we started this programme, we started on our own and with a little support from some NGOs. And now, everybody is speaking about building capacity, capacity building of local NGOs (Interview NGO Mitglied, 24. September 2012).

When we speak about empowering the judges, we started that initiative, now everybody is speaking about it, when we started with that in 2004, nobody cared, they [Internationals] only wanted to emphasize on women questions (Interview NGO Mitglied, 24. September 2012).

Die obigen Zitate deuten an, dass das Wissen um relevante Themen nicht nur auf Seiten der Geber verortet ist und auch nicht nur deren Wissen zum Tragen kommt. Gleichzeitig zeugen sie aber auf der Ebene von Machthierarchien in materiellen Austauschbeziehungen durchaus von einem binären Verständnis zwischen lokal und extern. Dennoch wird auch deutlich, dass sich Beziehungen als Teil des lokalen Raumes im Verlauf der Zeit wandeln und so auch den lokalen Raum prägend verändern. Entgegen dem weitläufigen Verständnis der FuK lassen sich die Beziehungen zwischen lokalen und externen Partnern also nicht nur auf ein einseitiges Machtverhältnis beschränken.

Obwohl Machtbeziehungen ganz überwiegend hierarchisch zugunsten der internationalen Geber strukturiert sind, verfügen auch lokale Organisationen über Macht in bestimmten Bereichen. Eine Mitarbeiterin einer europäischen NGO schildert im Gespräch, dass sie nicht nur bezüglich der Implementierung von Projekten, sondern auch bei der Suche nach neuen Projektpartnern auf die Aktivistinnen ihrer Partnerorganisationen angewiesen sind. Das explizite Ziel der Geberorganisation, auch Organisationen im ländlichen Raum zu fördern, lässt sich ohne die Mithilfe der Aktivistinnen kaum umsetzen, weil schlichtweg das Wissen über kleine NGOs in ländlichen Räumen fehlt und diese Gebiete für „Externe" häufig schwer zugänglich bleiben. In diesem Sinne verfügen hier lokale Organisationen durchaus über situatives Wissen - dies impliziert jedoch nicht, dass das situative Wissen dichotom zu anderen verstanden werden sollte. Denn ebendieses Wissen bewirkt, dass Machtbeziehungen zumindest stellenweise aufgebrochen werden, weil lokale Partnerorganisationen zeitweise mitbestimmen, mit welchen anderen Organisationen die Geber in Kontakt kommen. Die Perspektive internationaler Geber war nicht im Fokus des Projektes; dennoch deuten Gespräche auch hier darauf hin, dass Dichotomien weder einfach fortbestehen, noch gänzlich aufgebrochen werden. Auf der Ebene der Organisationen wird in Berichten und Gesprächen durchaus zwischen lokalen und internationalen Organisationen unterschieden (Kvinna till Kvinna, 2012; eigene Interviews). NGO-Mitarbeiterinnen, die von den jeweiligen internationalen Organisationen in den Irak entsandt sind, reflektieren Machtverhältnisse aber durchaus kritisch und sind bemüht, sie in der Zusammenarbeit mit Partnerorganisationen abzuschwächen - etwa in dem sie größtmögliche Selbstbestimmung zulassen und Aktivistinnen immer wieder dazu auffordern, eigene Belange deutlich nach außen zu kommunizieren.

Aus einer relationalen Perspektive gilt es weiterhin zu beachten, dass die Geberorganisationen selbst mittlerweile seit mindestens einem guten Jahrzehnt im Irak agieren und damit nicht mehr ohne weiteres ausschließlich als „extern“ beschrieben werden können. Dies wird besonders deutlich, wenn man die Heterogenität von Geber- und lokalen Organisationen und ihren Wissensformen einbezieht, die ich im nächsten Abschnitt analysieren möchte.

\subsection{Heterogenität vor Ort}

Die genannten Akteure selbst sind kaum in homogene Gruppen von lokal und extern einzuordnen. Ebenso weisen ihr Wissen und darauf basierende Handlungsansätze die unter- 
schiedlichsten Bezüge auf: Einige Momente in Wahrnehmung und Wirken der Akteure bestätigen Dichotomien von extern und intern, global und lokal; andere verweisen auf Brüche, die eine relationaleres Verständnis erfordern. Obwohl Aktivistinnen ihre Organisationen als lokal bezeichnen, leben und arbeiten sie in einer Umgebung, die stark durch internationales Engagement und globale Entwicklungen geprägt sind. Wie ich andernorts argumentiert habe, können NGOs eher als hybride Räume verstanden werden (Henrizi, 2015). Die Akteurinnen selbst sind zwar eng mit dem Irak und Bagdad im Speziellen verbunden, haben aber mehrheitlich auch Beziehungen zu westlichen Ländern (z.B. durch eigene Auslandsaufenthalte, Netzwerke, Verwandtschaftsbeziehungen, Workshops etc). Was im lokalen Raum passiert, ist also zunächst von Akteuren abhängig, deren Beziehungen sich weit über den geographischen Ort Bagdad bzw. Irak erstrecken. Weiterhin waren einige der Aktivistinnen selbst für internationale NGOs und Geberorganisationen tätig. Diese Faktoren tragen dazu bei, dass sich „lokales“ Wissen mit sog. ,Expertenwissen“ vermischt.

When you work with international organizations you get a lot of knowledge [on for example peacebuilding strategies], and when you work with the grassroots you know the reality so if someone can have both combinations, you are lucky (Interview NGO Mitglied, 8. April 2012).

Die Schilderung der Aktivistin beinhaltet zunächst ein dichotomes Bild von lokalem als situativem Wissen um lokale Gegebenheiten und von externem als Expertenwissen. Es ist aber nun gerade die Kombination aus beidem, die sie wertschätzt. Diesem Verständnis folgend liegen die Expertisen von lokalen Aktivistinnen und externen Akteurinnen durchaus in verschiedenen Bereichen: Während sich externes Wissen mehr auf inhaltliche Aspekte wie Gewalt gegen Frauen, Postkonfliktkontexte etc. bezieht, sind lokale Expertinnen in der Lage, lokale Spezifitäten ebendieser Themen auszumachen und Möglichkeiten aufzuzeigen, wie Projekte lokal sinnvoll implementiert werden können. Dazu können sie gerade aufgrund ihrer eigenen internen Heterogenität auf breite Bestände von kulturellem Wissen zurückgreifen. Außerdem sind sie durch ihr sprachliches Wissen in der Lage, überhaupt mit der Bevölkerung und Stakeholdern zu kommunizieren.

Dennoch sind Wissensbestände nicht derart eindeutig zu trennen; auch lokale Organisationen verfügen über Expertenwissen: So existiert beispielsweise auch auf lokaler Seite ein breiter Wissensvorrat über geschlechterbasierte Gewalt und - teilweise divergierende - Vorstellungen über Genderbeziehungen und gesellschaftliche Wandlungsprozesse. Gerade weil Aktivistinnen ihre Arbeit als (gesellschafts-)politisch begreifen, haben sie auch eigene Vorstellungen davon, wie sich etwa Genderbeziehungen im kulturellen Kontext des Irak verändern sollten, nämlich nicht unbedingt in Richtung einer Gleichheit zwischen Mann und Frau, sondern eher im Sinne einer gleichberechtigten Reziprozität (zu diesem Mo- dell von Genderbeziehungen für den arabischen Raum vgl. Winkel, 2012:169ff.). Eine Aktivistin, deren Karriere in einem Jugendprogramm einer lokalen Organisation begann, ist mittlerweile in verschiedenen Ländern als Genderexpertin einer großen amerikanischen Geberorganisation tätig; dennoch ist sie nebenberuflich weiterhin in der irakischen NGO Szene aktiv und führt so Wissen zurück. Es sind also auch heterogene, menschliche Austauschbeziehungen, welche die Konstitution des lokalen Raums prägen. Wie bereits erwähnt, haben sich einige Akteurinnen schon sehr lange innerhalb und außerhalb des Landes zivilgesellschaftlich - häufig mit Bezug auf Genderthemen - engagiert. Sie sind mittlerweile selbst Expertinnen auf diesem Gebiet. Lokales Wissen ist also nicht per se mit Laienwissen gleichzusetzen. Die Aktivistinnen verfügen eben nicht nur über situiertes Wissen, sondern sind auch in Bezug auf andere Inhalte häufig versiert. Der Anteil und Einfluss von „externer“ Expertise ist hier nicht genau nachvollziehbar, weil sich Inhalte vermischen und in der Wahrnehmung der Akteurinnen nicht mehr binär gegenüberstehen. Weder externes, noch lokales, Laienoder Expertenwissen existieren somit als abgrenzbare Phänomene in „Reinform“; vielmehr vermischen sie sich im Alltäglichen zu hybriden Wissens- und Handlungsformationen.

Heterogenität ist auch charakteristisch für die Einordnung externer Akteure aus Sicht der NGOs. Externe Akteure sind - aus der Perspektive der NGOs - kaum als homogene Gruppe zu betrachten. Ihre Akzeptanz ist stark von der Herkunft der Organisationen, ihrer Politik aber auch von individuellen Akteuren abhängig. So berichten Aktivistinnen, dass europäische Organisationen im Allgemeinen kooperativer arbeiten als solche aus den USA:

But they [American donor organisations] still have the problem of monitoring anybody, the Europeans they are much better, even previously, they are very sensitive in imposing agendas, they try to hear from the people (Interview NGO Mitglied, 24. September 2012).

Während einige Geberorganisationen Programme streng vorgeben (eigene Interviews), plant eine der europäischen Organisationen keine eigenen Projekte, sondern unterstützt ausschließlich lokale Organisationen in der Projektentwicklung. Projekte müssen zwar mit der allgemeinen inhaltlichen Ausrichtung der Organisation übereinstimmen und auch hier gibt es Vorstellungen seitens der Vertreterinnen der Geberorganisation; die Gestaltungsfreiheit lokaler Organisationen ist aber ungleich offener.

Außerdem wenden sich lokale NGOs - sofern die Wahl besteht - eher an Organisationen, von deren Arbeitsweise und inhaltlicher Orientierung sie am meisten überzeugt sind oder es größere Übereinstimmungen mit eigenen Handlungsansätzen gibt, ohne dass die geographische Verortung hier eine Rolle spielt. So berichtet eine Aktivistin, dass sie durchaus auch Anfragen internationaler Geber ablehnen, wenn diese nicht zu eigenen Prioritäten passen: „And if we go to donors, 
we can't do everything, they ask us for proposals [for certain projects], but we didn't sent it, because it is not in the strategy plan [of the NGO]" (Interview NGO Mitglied, 25. September 2012). Schließlich trägt auch die zeitliche Dimension des Wirkens internationaler Akteure dazu bei, dass Experten sich lokales Wissen aneignen und damit abermals Dichotomien durchbrechen. Viele Mitarbeiterinnen von Geberorganisationen arbeiten selbst seit vielen Jahren im Irak und haben enge Beziehungen zu ihren Partnerorganisationen aufgebaut und so ein tieferes Verständnis lokaler Gegebenheiten erlangt.

\subsection{Bewertung externer Akteure und Wissensformen durch lokale Akteure}

Externe Expertise wird von den interviewten Frauen insgesamt wesentlich differenzierter betrachtet, als es das Paradigma des ,schlechten Externen“ vorgibt. Meine Interviewpartnerinnen sehen externes Wissen - hier meist von internationalen Akteuren und NGOs vermittelt - und Strukturen (insbesondere internationale Verträge und Richtlinien) durchaus als hilfreich an. Teilweise existieren Allianzen mit Geberorganisationen, die vielfach über die gemeinsame Identität als Frauen (Aktivistinnen) funktionieren. Gleichzeitig ist Hilfe und Vermittlung von Erfahrungen oftmals erwünscht, aber eben in kooperativen Formen der Wissensanwendung und Projektimplementierung (Eigene Interviews). Schließlich werden externe Akteure und deren Agenden - insbesondere internationale Vereinbarungen und Verträge - auch als Mittel gewählt, um lokale Verhältnisse zu verändern. Die genannten Aspekte sollen im Folgenden beispielhaft veranschaulicht werden.

Trotz der Kritik der Aktivistinnen am internationalen Engagement im Irak, insbesondere der Verlauf des Sturzes Saddam Husseins und das Handeln nach der Invasion, betonen Aktivistinnen, dass internationale Hilfe notwendig war und ist, um die irakische Zivilgesellschaft zu reaktivieren.

After 35 years of suffering from dictatorship, he totally destroyed the civil society movement in Iraq... I mean since 1970 when Saddam started to destroy everything, and this was part of what he destroyed, and now we need to focus on building capacity for women (Interview NGO Mitglied, 24. September 2012).

We are always working with international organisations, and we feel that the support we got during the past 20 years was really good ... And we also believe it has given us not only financial [support] but always also experience ... and it was really wonderful to build our capacity (Interview NGO Mitglied, 24. September 2012).

Die Fähigkeit und Motivation sich zivilgesellschaftlich zu engagieren, gingen unter dem Regime verloren. Diktatur, Sanktionen und Kriege haben das Land völlig zerstört und
Misstrauen und Angst verbreitet, welche die sozialen Bande als Grundlage für eine funktionierende Zivilgesellschaft, aber auch den Aktivismus von Frauen quasi zerstörte. Internationale Hilfe ist aus dieser Perspektive aufgrund der Situation der irakischen Gesellschaft notwendig, die zumindest teilweise von Internationalen selbst bewirkt oder verschlimmert wurde. Aus dieser Perspektive ist das Lokale also nicht nur gut; auch im lokalen Raum finden sich problematische Tendenzen wie fehlendes Wissen und Kompetenzen, Misstrauen oder Rückzug in private Räume.

Das Fehlen von Einblicken und Erfahrungen in zivilgesellschaftlichem Engagement und in der Arbeit von Nichtregierungsorganisationen wird zum Teil durch Exilirakerinnen kompensiert, die sich diese Kompetenzen im Ausland aneignen konnten. Eine Aktivistin (die selbst nicht im Exil gelebt hat) schildert wie folgt:

I think the women who have been outside, they have a better knowledge of NGOs than those who were here, because they have their vision, they know about civil society, they learned the lesson (Interview NGO Mitglied, 10. Mai 2012).

Externe Expertise - ob von Externen vermittelt oder durch Erfahrungen im Ausland selbst gewonnen - wird also nicht per se als schlecht, sondern durchaus auch als hilfreich betrachtet. Die Dichotomie von gut-lokal und schlecht-extern wird hier überwunden; allerdings finden sich die Gegensätze von extern und lokal somit - zumindest ansatzweise - auch im Denken lokaler Aktivistinnen, wenn auch differenziert bewertet.

Externe Strukturen können darüber hinaus auch als Instrument genutzt werden, um - aus der Perspektive der Aktivistinnen - ,schlechtes“ Lokales zu verändern. So stellt etwa die CEDAW (Convention on the Elimination of All Forms of Discrimination Against Women)-Konvention einen wichtigen Kanal für Frauenorganisationen dar, um die irakische Politik zu beeinflussen. Die unterzeichnenden Staaten verpflichten sich, alle vier Jahre über die Situation von Frauen in ihrem Land und Fortschritte beim Abbau von Diskriminierung zu berichten. NGOs haben die Möglichkeit, einen alternativen Bericht, den sog. „Shadow Report“, bei der Kommission einzureichen und so den staatlichen Bericht sowie staatliche Maßnahmen zu bewerten bzw. zu kritisieren. Obwohl das irakische Recht weitgehend im Einklang mit CEDAW ist, bleibt die lückenhafte oder fehlende Durchsetzung des Rechts ein großes Problem. Das internationale Reglement ermöglichte, den Staat zumindest mit dem Problem zu konfrontieren und bestenfalls auch Änderungen im lokalen Raum herbeizuführen. Darüber hinaus dient Lobbyarbeit im Rahmen der CEDAW-Konvention aus der Perspektive der Aktivistinnen dazu, Wissenslücken und Ignoranz gegenüber der Diskriminierung von Frauen in der irakischen Bevölkerung entgegenzuwirken oder mit lokalen Wissensbeständen $\mathrm{zu}$ verknüpfen (Eigene Interviews und teilnehmende Beobachtung 2012-2014). Abgesehen von einer Bewertung in gut 
und schlecht bleibt offen, inwiefern CEDAW überhaupt nur als „globales“ Instrument gesehen wird. Gerade aus einer raumtheoretischen Perspektive ist CEDAW eher als translokal denn als global bezeichnet worden, schließlich ist die Konvention in einem lokalen (westlichen) Kontext entstanden und nur auf lokaler Ebene wirksam (Zwingel, 2012; Henrizi, 2016).

Schließlich können aus der Perspektive der Aktivistinnen auch externe Akteure - wie z.B. die UN-Organisationen gute Arbeit leisten; allerdings bestenfalls in Zusammenarbeit mit lokalen Akteuren. Einige meiner Interviewpartnerinnen haben sich zeitweise bewusst für die Arbeit bei internationalen Organisationen entschieden, um sich deren Expertise (z.B. im Bezug auf Projektmanagement oder Peacebuilding) anzueignen und gleichzeitig ihre eigenen Vorstellungen in die Organisationen zu tragen. Letzteres umfasst nun aber eben gerade nicht nur das situierte Wissen lokaler Akteure, sondern konkrete Vorstellungen über die inhaltliche Ausrichtung der internationalen Organisationen. Gerade in dieser Hinsicht wird deutlich, dass die interviewten Frauen längst selbst über „Expertinnenwissen“ verfügen und sich ihr Wissen kaum auf Kenntnisse des lokalen Orts begrenzen lässt.

\section{Ambivalenzen des lokal-global-Paradigmas - Synthese und Fazit}

Ich habe in meiner empirischen Analyse gezeigt, dass dichotome Vorstellungen von lokalem Wissen als „,gutem“, situiertem bzw. Laienwissen und externem als ,schlechtem“ Expertenwissen zu kurz greifen, möchte man die Wissensformationen in der Gesellschaft des Irak in ihrer vielfältigen Hybridität nachvollziehen: Lokal und global sind hier äußerst ambivalente Konzepte: Aus der Perspektive von Frauenaktivistinnen gestalten sich die Beziehungen zu internationalen Akteuren und die Wahrnehmung externer Expertisen wesentlich heterogener als ein solches Verständnis zulässt. Obwohl Dichotomien nicht nur in der Theorie, sondern auch in den Köpfen der Akteurinnen vor Ort fortbestehen, werden sie im alltäglichen Handeln immer wieder auch durchbrochen und durchkreuzt.

Die Vermischung von Wissen zu hybriden Formationen und Projekten hat zur Folge, dass die Zuordnung von lokalem vs. externem Wissen nicht immer möglich und sinnvoll ist: In Gesprächen ist häufig nicht eindeutig nachvollziehbar, von wem bestimmte Ideen oder Ansätze initiiert wurden. So machte eine Vertreterin einer amerikanischen Organisation im Gespräch deutlich, dass Projekte hauptsächlich von ihnen initiiert werden und lokale Partner soweit wie möglich für die Implementierung hinzugezogen werden. Eine Vertreterin einer lokalen NGO erzählte jedoch im Interview mit Bezug auf ebendiese Projekte, es seien ,ihre“ Ideen gewesen (Eigene Interviews). Unabhängig davon, welchen Anteil die lokale Organisation tatsächlich an der Einrichtung hatte, ist der An- spruch auf bzw. das Postulieren von Gestaltungsmöglichkeiten hier von Bedeutung. Für das Selbstverständnis und die Motivation der Aktivistinnen ist es wichtig, Ideen als ,ihre lokalen“, aber auch als Expertisen zu postulieren und anzunehmen. Die Ideen als „lokal“ zu bezeichnen steht hier weniger im Zusammenhang mit dem geographischen Ort, sondern dient vielmehr der Legitimation eigenen Handelns (vgl. dazu Haughton et al., 2015). Nicht nur die Zuordnung von Wissen, sondern die Verwendung des Attributs „lokal“ seitens der Aktivistinnen ist selbst schon ambivalent. Expertise bleibt immer auch ein Prozess der Zuschreibung und der Anerkennung von Wissen.

Besonders eindrücklich sind Ambivalenzen in Bezug auf Wissensbestände zu Genderthemen: Obwohl die untersuchten NGOs sich in ihrer Arbeit internationaler Definitionen, Konzepte und Verträge (wie etwa CEDAW oder die UNResolution 1325 zum Schutz von Frauen in Konflikten) bedienen und eng mit Geberorganisationen kooperieren, ist es - insbesondere auch auf Grund der Kritik aus der eigenen Bevölkerung - von immenser Bedeutung, Frauenrechte und Engagement von Frauen als etwas inhärent irakisches zu positionieren. Die oben geschilderten Auswirkungen externer Ziele und Handlungsansätze auf den lokalen Kontext werden in diesem Bereich besonders auf diskursiver Ebene deutlich. Insbesondere die Rhetorik der Alliierten im Vorfeld der Invasion, die irakischen Frauen ,retten“ zu wollen hat dazu geführt, dass Frauenrechte und Genderthemen in der irakischen Bevölkerung und Politik häufig negativ - als Besatzeragenda - konnotiert sind. Aktivistinnen bemühen sich, dem entgegenzuwirken, indem sie auf die historische Stellung der Frau und die lange Tradition der Frauenbewegungen im Irak hinweisen. Dennoch sind sie bedingt durch das - vermeintlich gutgemeinte - Handeln internationaler Akteure immer wieder dem Vorwurf der „Verwestlichung“ ausgesetzt.

... the propaganda that it [gender] is western tendencies, it is false. ... When we speak about Gender we take from the Koran itself and we try to link it with Gender. In Koran it is very wonderful, when we speak about women and men together, on everything. Women and men, women and men. ... So this is very very obviously. When we speak to them [religious leaders] gender sensitivity it is really, that we always make. Always we make this, look this is the Koran, so why are we not speaking about it, we are using it in a very wonderful way. We convince them (Interview NGO Mitglied, 24. September 2012).

Während Gender in Teilen der irakischen Gesellschaft als „westlich“ verpönt wird, versuchen Aktivistinnen es als universales Thema zu proklamieren, dass eben nicht westlich ist, sondern sich auch im Koran wiederfindet, der universell ist und gleichzeitig einen regionalen Bezug hat und hohe kulturelle Autorität genießt. Die Strategie ist hier weniger als persönliche Überzeugung (sie selbst ist Christin) denn als Reak- 
tion auf den irakischen Kontext zu verstehen, in dem immer wieder die Dichotomie zwischen Gender/Westen und Islam politisiert wird.

Jenseits der Ambivalenzen der Konzepte von lokal und global und damit verbundenen Spannungen zwischen Akteuren und Wissensbeständen bleibt das Potential hybrider Wissensformationen zu betonen: Haughton et al. (2015) schildern die Vereinbarung von lokalem und sogenanntem Expertenwissen als innovative Lösung die im Management von Flutrisiken angewendet wird. Jenseits von romantisierten Vorstellungen solcher Kooperationen, sind es ebensolche hybriden Formen des Wissens und seiner Anwendung, die sich in der Praxis wiederfinden. Die Analyse aus der Perspektive des Lokalen wirft gleichwohl die Frage auf, inwieweit die Konfliktlinie ,extern vs. lokal“ mit anderen Abgrenzungen und Dichotomien verschränkt ist. Aus den Daten wird deutlich, dass sie nur eine unter vielen - etwa MannFrau, Zivilgesellschaft-Politik, gesellschaftliche Gruppierungen - darstellt. Eine weitere Analyse ebensolcher Verschränkungen könnte zukünftig dazu dienen, binäre Abgrenzungen zwischen extern und lokal weiter aufzubrechen.

Die Diskurse hinken - zumindest in der FuK - der Realität häufig hinterher, indem sie weiter an binären Konzepten festhalten. Meine empirischen Daten laden dazu sein, lokale Räume und Akteure in ihrer Heterogenität zu begreifen; d.h. aber gerade auch, sie nicht zu romantisieren. Ein relationales Raumverständnis hilft, die Relevanz von Akteuren für die komplexe Konstitution des lokalen Raums systematisch anzuerkennen und Dichotomien aufzubrechen bzw. dort sichtbar zu machen, wo sie fortbestehen. Die Herausforderung liegt hier darin, die Ambivalenz zwischen Fortbestehen von Dichotomien in Theorie und Praxis und ihre gleichzeitige Auflösung im alltäglichen Handeln auszuhalten. In Anbetracht der vielfältigen Subjektpositionen, die das Geflecht aus Beziehungen und Netzwerken prägen stellt sich auch die Frage, inwieweit konzeptionelle Zuordnungen von ,extern“ und ,lokal“ überhaupt sinnvoll sind bzw. inwiefern sie dennoch auch notwendig bleiben, um Akteure und Wissensbestände überhaupt benennen zu können.

\section{7 Überblick 1: Untersuchte externe und lokale Akteursgruppen}

Meine Forschung hat sich in erster Linie auf Aktivistinnen in lokalen Nichtregierungsorganisationen konzentriert. Alle anderen Akteursgruppen wurden (abgesehen von einigen wenigen Experteninterviews) nur durch die Brille der Frauenaktivistinnen analysiert; außerdem habe ich für den historischen Teil zusätzlich auf die zitierte Sekundärliteratur zurückgegriffen.

Folgende Organisationen/Institutionen waren für die jeweiligen Bereiche relevant:
1. Lokale Akteure:

a. (Frauen)organisationen im Zentrum der Analyse, z.B. Al Amal, Baghdad Women's Organization, Iraqi Women's League

b. Andere Lokale Akteure wie z.B. religiöse Führer, Stammesführer, kommunalpolitische Akteure

2. Externe Akteure:

c. Internationale Organisationen (insbesondere der UN zugehörig) wie UNAMI, UNWOMEN, UNOPS

d. Internationale Nichtregierungsorganisationen einzelner westlicher Staaten oder staatsübergreifend wie Norwegian People's Aid, Kvinna till Kvinna, Mercy Corps, International Rescue Committee, Oxfam

e. Regierungen anderer Staaten

\section{Datenverfügbarkeit}

Die verwendeten Daten sind nicht öffentlich verfügbar. Weitere Informationen können bei der Autorin erfragt werden.

Interessenkonflikt. Die Autorin erklärt, dass kein Interessenkonflikt besteht.

Danksagung. Ich danke den irakischen Frauen, die meine Forschung unterstützt und ermöglicht haben, in dem sie mich an ihrer Arbeit teilhaben ließen. Weiterhin möchte ich mich bei den drei anonymen Reviewer_innen und der Herausgeberin Anne Vogelpohl für die konstruktive Kritik und zahlreiche Anregungen bedanken sowie bei der Studienstiftung des deutschen Volkes, die mir die Forschung ermöglicht hat.

Edited by: A. Vogelpohl

Reviewed by: three anonymous referees

\section{Literatur}

Ackerly, B., Stern, M. und True, J.: Feminist Methodologies for International Relations, Cambridge University Press, Cambridge, 2006.

Al-Ali, N.: Gender and Civil Society in the Middle East, International Feminist Journal of Politics, 5, 216-232, 2003.

Al-Ali, N.: Reconstructing Gender: Iraqi women between dictatorship, war, sanctions and occupation, in: Reconstructing PostSaddam Iraq, Herausgeber: Barakat, S., Routledge, New York, 2008.

Al-Ali, N. und Pratt, N.: What Kind of Liberation? Women and the Occupation of Iraq, University of California Press, Berkeley, 2009.

Al-Jawaheri, Y.: Women in Iraq: The Gender Impact of International Sanctions, Lynne Rienner Publishers, Boulder, 2008. 
Baram, A., Rohde, A. und Zeidel, R.: Introduction: Iraq: History Reconsidered, Present Reassessed, in: Iraq Between Occupations. Perspectives from 1920 to the Present, Herausgeber: Baram A., Rohde, A. und Zeidel, R. Palgrave, London, 1-12, 2010.

Björkdahl, A. und Buckley-Zistel, S. (Hrsg.): Spatializing Peace and Conflict. Mapping the Production of Places. Sites and Scales of Violence, Palgrave, London, 2016.

Buckley-Zistel, S.: Frictional Spaces: Transitional Justice between the Global and the Local, in: Peacebuilding and Friction. Global and Local Encounters in Post-Conflict Societies, Herausgeber: Björkdahl, A., Höglund, K., Millar, G., van der Lijn, J. und Verkoren, W., Routledge, Abingdon, 17-31, 2016.

Charmaz, K.: Constructing Grounded Theory: A Practical Guide Through Qualitative Analysis, Sage, London, 2006.

Coyne, H.: Amateur Hour in Iraq. A Worm's-Eye View on the Failure of Nation Building, in: Iraq Between Occupations. Perspectives from 1920 to the Present, Herausgeber: Baram A., Rohde, A. und Zeidel, R., Palgrave, London, 225-237, 2010.

Cubitt, C.: Constructing civil society: an intervention for building peace?, Peacebuilding, 1, 91-108, 2013.

Dodge, T.: Inventing Iraq. The Failure of Nation Building and a History Denied, Columbia University Press, New York, 2003.

Efrati, N.: Back to Square One. Women's Rights in Post-Invasion Iraq, in: Post-Saddam Iraq. New Realities, Old Identities, Changing Patterns, Herausgeber: Cohen, A. und Efrati, N., Sussex Academic Press, Brighton \& Eastbourne, 171-186, 2011.

Fürtig, H.: Kleine Geschichte des Irak, Beck, München, 2004.

Fürtig, H.: Irak: Regimewechsel im Zweistromland, in: Einsatz für den Frieden. Sicherheit und Entwicklung in Räumen begrenzter Staatlichkeit, Herausgeber: Braml, J., Risse, T. und Sandschneider, E., Oldenbourg, München, 129-136, 2010.

Harvey, D.: Spaces of Neoliberalization: Towards a Theory of Uneven Geographical Development, Hettner Lecture 2004, Stuttgart, Steiner, 2005.

Harvey, D.: Cosmopolitanism and the Geographies of Freedom, New York, Columbia University Press, 2009.

Haughton, G., Bankoff, G. und Coulthard, T. J.: In search of "lost" knowledge and outsourced expertise in flood risk management, T. I. Brit. Geogr., 40, 375-386, 2015.

Hellmüller, S. und Santschi, M. (Hrsg.): Is Local Beautiful? Peacebuilding between International Interventions and Locally Led Initiatives, Springer, London, 2014.

Henrizi, A.: Building peace in hybrid spaces. Women's agency in Iraqi NGO's, Peacebuilding, 3, 75-89, 2015.

Henrizi, A.: Local Agency in "global" spaces? The engagement of Iraqi women's NGOs with CEDAW, in: Spatialising Peace and Conflict. Mapping the Production of Places, Sites and Scales of Violence, Herausgeber: Björkdahl, A. und Buckley-Zistel, Basingstoke, Palgrave, 2016.

Isakhan, B. (Hrsg.): The legacy of Iraq. From the 2003 to the Islamic State, Edinburgh University Press, Edinburgh, 2014.

Jabri, V.: War, government, politics: A critical response to the hegemony of the liberal peace, in: Palgrave Advances in Peacebuilding. Critical Developments and Approaches, Herausgeber: Richmond, O., Palgrave, Basingstoke, 41-57, 2010.

Jawad, Y.: The Plight of Iraqi Women 10 years of suffering, The 10th commemoration of the invasion and occupation of Iraq, Geneva International Center for Justice, Geneva, 14. und 15. März 2013, online aufrufbar: http://www.gicj.org/iraq_
conference_speeches/Dr_Yasmin_Jawad_Presentation.pdf (letzter Zugriff: 28. Oktober 2016), 2013.

Kamp, M.: Geschlecht, Kolonialismus und Nation. Patriarchale Nachkriegsordnung (en) im Irak, in: Frieden - Gewalt - Geschlecht. FuK als Geschlechterforschung, Herausgeber: Davy, A., Hagemann, J. und Kätzel, U., Klartext, Essen, 293-314, 2005.

Kemper, J. und Vogelpohl, A.: „Eigenlogik der Städte“? Kritische Anmerkungen zu einer Forschungsperspektive, in: Lokalistische Stadtforschung, kulturalisierte Städte - Zur Kritik einer „Eigenlogik der Städte“, Herausgeber: Kemper, J. und Vogelpohl, A., Münster: Westfälisches Dampfboot, 15-38, 2011.

Kbashi, A., Al Khateeb, B., Putros, M., Edwar, H. und Hammoud, H.: 2014 Civil Society Monitoring Country Report, Unpublished NGO Report, 2014.

Kvinna till Kvinna: Equal Power - Lasting Peace Obstacles for women's participation in peace processes, online aufrufbar: http://kvinnatillkvinna.se/en/files/qbank/ df0aab058ce179e4f7ab135ed4e641a9.pdf (letzter Zugriff 18. Oktober 2016), 2012.

Lefebvre, H.: The Production of Space, Oxford, Blackwell Publishing, 1991.

MacGinty, R.: Indigenous Peace-Making Versus the Liberal Peace, Coop. Confl., 43, 139-163, 2008.

MacGinty, R.: International Peacebuilding and Local Resistance. Hybrid Forms of Peace, Palgrave, Basingstoke, 2011.

MacGinty, R. und Richmond, O.: The Local Turn in Peace Building: a critical agenda for peace, Third World Q., 34, 763-783, 2013.

Massey, D.: A Global Sense of Place, Marxism Today 1991 (June), 24-29, 1991a.

Massey, D.: The Political Place of Locality Studies, Environ. Plann. A, 23, 267-281, 1991b.

Massey, D.: Space, Place and Gender Cambridge, Polity Press, 1994.

Massey, D.: Power-Geometries and the Politics of Space-Time, Hettner-Lecture 1998, Stuttgart, Franz Steiner, 1999.

Massey, D.: Geographies of responsibility, Geogr. Ann. B, 86, 5-18, 2004.

Peake, L. und Trotz, A.: Gender, Ethnicity and Place: Women and Identities in Guyana, London, Routledge, 1999.

Pierce, J., Martin, D. G. und Murphy, J. T.: Relational Place-Making - The Networked Politics of Place, T. I. Brit. Geogr., 36, 54-70, 2010.

Pouligny, B.: Civil Society and Post-Conflict Peacebuilding: Ambiguities of International Programmes Aimed at Building "New" Societies', Secur. Dialogue, 36, 495-510, 2005.

Porter, E.: Peacebuilding. Women in International Perspective, Routledge, New York, 2007.

Pugh, M.: The problem-solving and critical paradigms', in: Handbook of Peacebuilding, Herausgeber: MacGinty, R., Routledge, London, 11-24, 2013.

Rahbek, B.: Introduction: Dilemmas of Demcratisation in the Middle East, in: Democratisation in the Middle East: dilemmas and perspectives, Herausgeber: Rahbek, M., Aarhus University Press, Aarhus, 7-18, 2005.

Richmond O.: Becoming liberal, unbecoming liberalism: Liberallocal hybridity via the everyday as a response to the paradoxes of liberal peacebuilding, Journal of Intervention and Statebuilding, 3, 324-344, 2009. 
Richmond, O. (Hrsg.): Palgrave Advances in Peacebuilding. Critical Developments and Approaches, Palgrave, Basingstoke, 2010.

Richmond, O.: Beyond local: ownership and participation in the architecture of international peacebuilding, Ethnopolitics, 11, 354375, 2012.

Rohde, A: State-Society Relations in Ba'thist Iraq: Facing Dictatorship, London, Routledge, 2010.

Rohde, A. und Fischer-Tahir, A.: Introductory remarks to the conference: Gender in Iraqi Studies: Trans_local Actors and Knowledge Flows in Comparative Perspective, Marburg, 6. Mai 2015.

Shadow Report: Iraqi Women in Armed Conflict and Post Conflict Situation, Shadow Report submitted to the CEDAW Committee at the 57th Session, 2014.

Slash, A. und Tom, P.: Is liberal democracy possible in Iraq? in: Rethinking the Liberal Peace: External Models and Local Alternatives, Herausgeber: Tadjbakhsh, S., Routledge, Oxon, 195205, 2011

Streicher, R.: Die Macht des Feldes. Für Selbstreflexivität als methodologische Strategie feministischer Feldforschung in Konfliktgebieten, in Geschlechterverhältnisse, Frieden und Konflikt. Feministische Denkanstöße für die FuK, Herausgeber: Engels, B. und Gayer, C., 65-77, 2011.
Sultana, F.: Reflexivity, Positionality and Participatory Ethics: Negotiating Fieldwork Dilemmas in International Research, ACME, An International Journal for Critical Geographies, 6, 376385, 2007.

Swyngedouw, E.: Neither Global nor Local: "Glocalization" and the Politics of Scale, in: Spaces of globalization, reasserting the power of the local, Herausgeber: Kevin R. C., The Guilford Press, New York/London, 137-166, 1997.

Winkel, H.: Gender Knowledge in the Arabic-Islamic Realm, in: Gendered Ways of Knowing in Science, Herausgeber: Knaus, S., Wobbe, T. und Covi, G, Trento, FBK Press, 155-176, 2012.

Zwingel, S.: How Do Norms Travel? Theorizing international women's rights in transnational perspective, Int. Stud. Quart., 56, $115-129,2012$. 